\title{
EFFECT OF ROASTING TEMPERATURE ON THE MAGNETISM OF ILMENITE
}

\author{
N.R. ALLEN \\ Ka Pty Ltd, 14 Station Lane, Exton, Tasmania 7303, Australia
}

(Received 26 February 2003; In final form 4 March 2003)

\begin{abstract}
The enhancement of ilmenite magnetic susceptibility by roasting is well known, and it is used routinely in the magnetic separation of mineral sands, yet surprisingly little appears to be known in detail about the development of this magnetisation as roast temperatures increase. An examination of the changes in both magnetic susceptibility and magnetic rotatability within the roast temperature range $350-650^{\circ} \mathrm{C}$ reveals some interesting low-temperature magnetisation changes. The results suggest the presence at low roast temperatures of very small magnetised regions that behave as though they are superparamagnetic, and suggest that the growth of these regions plays a significant part in the development of roast-induced magnetisation. The results also show that the bulk magnetic development above $500^{\circ} \mathrm{C}$ is a resultant of several temperature-dependent competing processes.
\end{abstract}

Keywords: Magnetising roast; Ilmenite; Magnetic separation; RMF

\section{INTRODUCTION}

The experimental work described here arose from a need to determine optimum ilmenite roast conditions for the separation of roasted ilmenite by rotating magnetic field [1]. A series of test roasts designed to compare RMF rotation separation and conventional magnetic separation demonstrated that the roast conditions are different for the two magnetic separation methods, but did not determine optimum conditions for RMF rotation separation. Attempts to theoretically estimate optimum roast conditions for the enhancement of particle rotation, on the basis of known details of magnetic changes due to roasting, quickly discovered that 'known details' were surprisingly few in number, especially for roast temperatures below $650^{\circ} \mathrm{C}$.

The tests here only examine the magnetising effects of fluid bed oxidising (natural air) roasting, and form the first part of a planned more extensive investigation.

Note, that the unit for magnetic field, $H$, is the $\mathrm{A} / \mathrm{m}$. Throughout the discussion below, the more familiar unit $\mathrm{T}$ has been used. The $\mathrm{T}\left(\right.$ or $\left.\mathrm{Wb} / \mathrm{m}^{2}\right)$ is the unit of magnetic

E-mail: nallen@vision.net.au 
induction, $B$, and is related to the $\mathrm{A} / \mathrm{m}$ in free space (not in a magnetised material) by:

$$
B=\mu_{0} H
$$

where $\mu_{0}=4 \pi \times 10^{-7} \mathrm{~Wb} / \mathrm{Am}$.

In this article, a value has also been calculated for the fractional 'magnetic susceptibility'. This calculation has assumed that the particles are paramagnetic, which is not correct for all the particles here. Perhaps most of the unroasted particles will be basically paramagnetic, but as the roast temperature increases this number will reduce to a small minority. The 'susceptibility' values given or referred to here are mass susceptibilities (units of $\mathrm{m}^{3} / \mathrm{kg}$ ) and, as illustrated, should be taken only as an indication of relative particle 'attractability'.

\section{EXPERIMENTAL METHODS}

\subsection{Roasting}

The ilmenite sample, with a particle size range from $75 \mu \mathrm{m}$ to $200 \mu \mathrm{m}$, came from the Murray Basin, Australia, and had already been through a wet high-intensity magnetic separation (WHIMS). Sample roasting was carried out, on between 80 and $100 \mathrm{~g}$ of the sample at a time, using the small heat-exchange roaster shown in Fig. 1. The sample container, including the pyrometer, can be lifted off to transfer the sample to cooling containers after roasting. This roaster has an upper roasting temperature of just above $650^{\circ} \mathrm{C}$.

Three roasts $(15,30$ and $60 \mathrm{~min})$ were carried out at each roast temperature from 350 to $650^{\circ} \mathrm{C}$, at intervals of $50^{\circ} \mathrm{C}$. Roast temperatures were measured within the fluidised sample, and were recorded at 1 min intervals throughout the roasting. The roast time included the time for the sample to heat up to roast temperature (a few minutes). Immediately after roasting, half the sample was allowed to cool in the absence of a magnetic field, while the other half was cooled in a static magnetic field. The magnetic field was provided by ceramic magnets arranged on either side of a stainless steel cooling trough. The magnetic field in the cooling trough varied from $0.13 \mathrm{~T}$ up to almost $0.18 \mathrm{~T}$, depending on location between the magnets. Field measurements after each

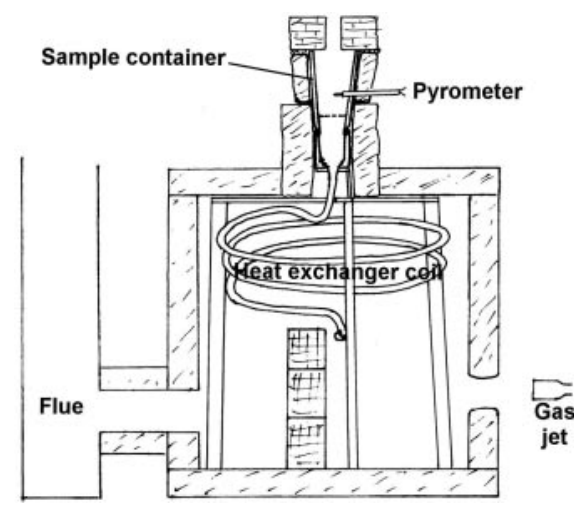

FIGURE 1 Sketch of heat-exchange fluid-bed roaster. 
roast showed no measurable field degrading caused by heat effects on the magnets. This simple method of cooling in a magnetic field was only intended to give a preliminary indication of the effects.

\subsection{Magnetic Separation}

All magnetic separations were carried out on the Ka RMF laboratory separator shown in Fig. 2. This separator can carry out both the RMF lift separations and the RMF particle rotation separations. RMF lift separation is a conventional particle attraction separation, with the particle rotation being used during separation to lower entrapment.

RMF lift magnetic separations were carried out, with the separator set up as shown in Fig. 2, at lift distances commencing at $45 \mathrm{~mm}$ below the magnet rotor, and then at $5 \mathrm{~mm}$ intervals until $5 \mathrm{~mm}$ below the magnet rotor. A mass magnetic susceptibility was calculated for each lift distance [2], allowing the sample distributions to be expressed in terms of a calculated magnetic susceptibility. The magnetic field and field gradient at each lift distance is given in Table I.

The lift separations were carried out in batches of six, for each roast temperature, before commencing the rotation separations. This was done to minimise configuration changes to the separator that may have accidentally altered settings. The lift separations were done first to delay exposing the sample to the higher $(0.4 \mathrm{~T})$ rotation separation field before all the lift separations (maximum $0.36 \mathrm{~T}$ at $5 \mathrm{~mm}$ lift) were completed.

The concept of particle rotation separation is illustrated in Fig. 3. The lowest three fractions from the lift separations (10 mm lift down to non-mags) were re-combined and the separator converted to the RMF rotation separation. This conversion entailed raising the feeder to feed above the magnet rotor, and swinging the feeder mechanism around $180^{\circ}$ to feed from the left. The splitter shown in Fig. 3 allows the rotating

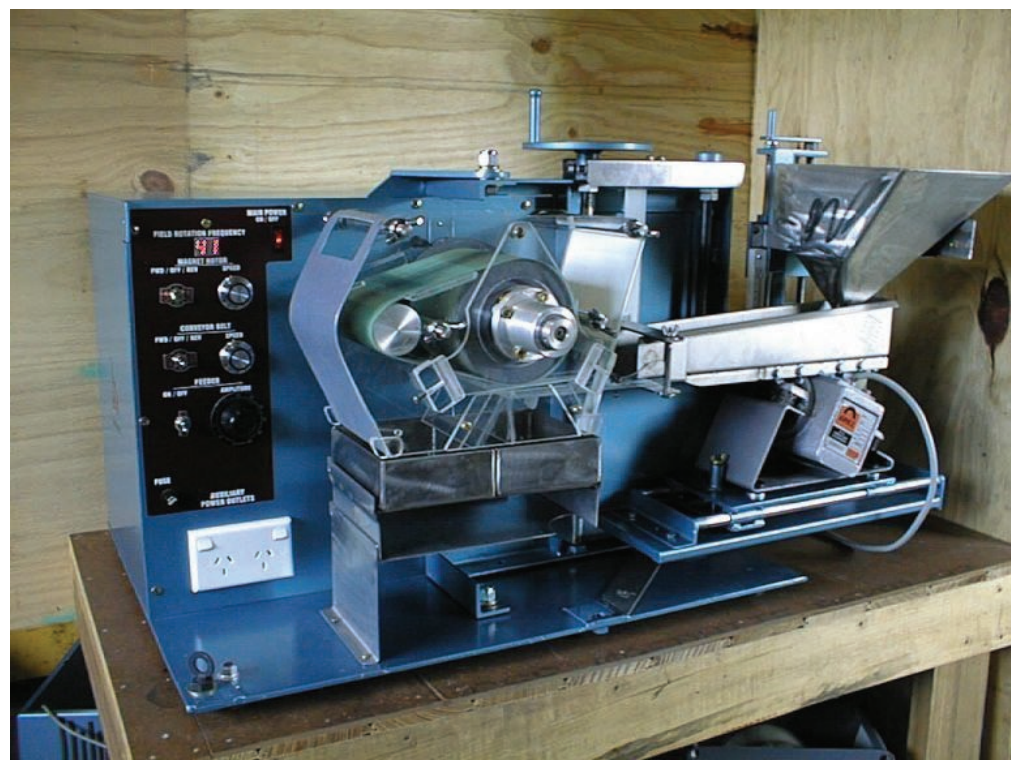

FIGURE 2 The Ka RMF laboratory separator, set up for RMF lift separation. For rotation separation the feeder can be rotated around to feed from the left and above the magnet rotor. 
TABLE I Magnetic fields and field gradients at RMF separation lift distances

\begin{tabular}{lcc}
\hline Lift distance $(\mathrm{mm})$ & Field $(\mathrm{T})$ & Field gradient $(\mathrm{T} / \mathrm{m})$ \\
\hline 45 & 0.005 & 0.52 \\
40 & 0.008 & 0.89 \\
35 & 0.014 & 1.52 \\
30 & 0.024 & 2.61 \\
25 & 0.042 & 4.46 \\
20 & 0.071 & 7.64 \\
15 & 0.122 & 13.07 \\
10 & 0.208 & 22.38 \\
5 & 0.356 & 38.31 \\
\hline
\end{tabular}

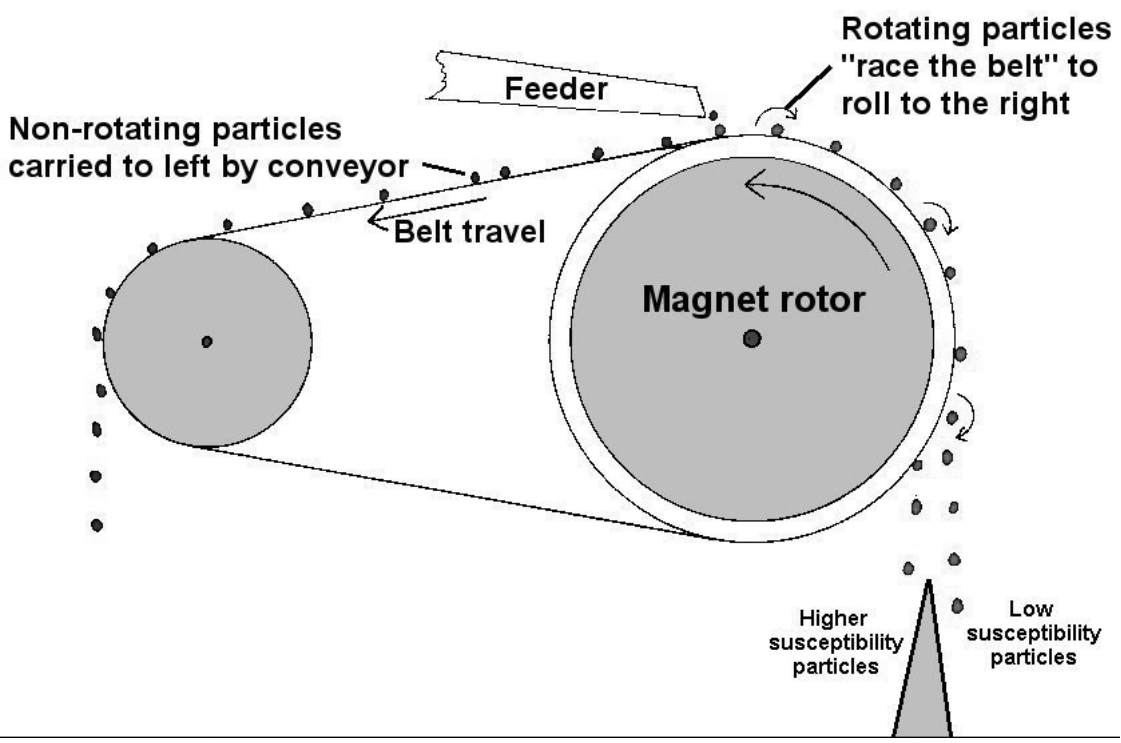

FIGURE 3 The concept of RMF rotation separation. The belt speed is set at approximately half the average particle rolling speed.

particles to be divided into two fractions on the basis of magnetic susceptibility so that the rotation separation produced three magnetic fractions. This was found necessary in mineral sands separations because the low susceptibility rotation fraction ('rot-low-mags' in the figures) often contains high chrome values. The exact setting for this splitter would normally be determined with reference to actual fraction $\mathrm{Cr}_{2} \mathrm{O}_{3}$ assay values for a given sample. In this case it was set at an 'average' position, based on past experience.

\subsection{Initial Demagnetisation Test}

The ilmenite sample used for these roasting tests had, as a part of its earlier processing, been through a wet high intensity magnetic separation (WHIMS). In a mineral sands operation this would be a normal pre-roast process, and could be expected to leave some magnetic remanence in the sample. In order to check on this, one unroasted sample was passed through a simple demagnetisation process and then magnetically 
separated as described above. The demagnetisation involved confining the sample between the separator conveyor belt in Fig. 3 and a stretched plastic sheet (to stop any particle rotation), and then, with a $0.4 \mathrm{~T}$ field rotation at $170 \mathrm{~Hz}$, moving the conveyor belt anti-clockwise to slowly move the sample out of the magnetic field and subject it to a gradually decreasing rotating field strength. The $0.4 \mathrm{~T}$ field used for this purpose is about $0.04 \mathrm{~T}$ higher than the field for the $5 \mathrm{~mm}$ lift separation.

\section{RESULTS}

The lift separation results have been plotted in the figures that follow as frequency distributions with the class intervals being defined by the lift distances. The rotation separation results have been plotted in only five 'intervals'. The $+25 \mathrm{~mm}$ lift fractions includes all fractions from $25 \mathrm{~mm}$ lift and above. These would often be rejected in a mineral sands separation because of high chrome values. The 15 and $20 \mathrm{~mm}$ lift fractions have been combined. Almost all these particles will rotate strongly, and would normally be lift separated prior to a rotation separation in order to avoid particlerelease problems under the magnet rotor. The separation fraction percentages for the unroasted sample and the 350,400 and $450^{\circ} \mathrm{C}$ roasts are given in Tables II-V.

\section{1. $350^{\circ} \mathrm{C}$}

The $350^{\circ} \mathrm{C}$ roast produced results (Table III and Fig. 4) that may initially seem puzzling. There was a significant increase in the non-magnetic susceptibility fraction (not lifted at $5 \mathrm{~mm}$ ), indicating that many particles had decreased in susceptibility. At the same time, an increase in the $10 \mathrm{~mm}$ lift and $15 \mathrm{~mm}$ lift fractions occurred, indicating that susceptibility had increased for some other particles.

TABLE II Separation results for the unroasted sample

\begin{tabular}{lcc}
\hline \multicolumn{3}{c}{ RMF lift separation } \\
\hline RMF lift distance $(\mathrm{mm})$ & $\begin{array}{c}\text { Average magnetic } \\
\text { susceptibility }\left(\mathrm{m}^{3} / \mathrm{kg}\right)\end{array}$ & Separated weight $(\%)$ \\
\hline 45 & $4.9 \times 10^{-3}$ & 0.01 \\
40 & $1.7 \times 10^{-3}$ & 0.02 \\
35 & $5.7 \times 10^{-4}$ & 0.02 \\
30 & $2.0 \times 10^{-4}$ & 0.1 \\
25 & $6.7 \times 10^{-5}$ & 0.43 \\
20 & $2.3 \times 10^{-5}$ & 1.78 \\
15 & $7.7 \times 10^{-6}$ & 6.75 \\
10 & $2.6 \times 10^{-6}$ & 16.99 \\
5 & $9.0 \times 10^{-7}$ & 50.09 \\
Non-mag & $4.7 \times 10^{-7}($ est.) & 23.82 \\
\hline & Rotation separation & \\
\hline Fraction & & Separated weight $(\%)$ \\
\hline+25 mm RMF lift & & 0.58 \\
15-25 mm RMF lift & & 8.53 \\
Rotating & & 24.71 \\
Rotate-low mag split & & 8.88 \\
Non-rotating & & 56.74 \\
\hline
\end{tabular}


TABLE III Separation results for the $350^{\circ} \mathrm{C}$ roast

\begin{tabular}{|c|c|c|c|c|c|c|}
\hline \multicolumn{7}{|c|}{ RMF lift separation } \\
\hline \multirow[t]{3}{*}{ Separation distance $(\mathrm{mm})$} & \multicolumn{6}{|c|}{ Separated weight percent } \\
\hline & \multicolumn{3}{|c|}{ With magnetic treatment } & \multicolumn{3}{|c|}{ No magnetic treatment } \\
\hline & $15 \mathrm{~min}$ & $30 \mathrm{~min}$ & $60 \mathrm{~min}$ & $15 \mathrm{~min}$ & $30 \mathrm{~min}$ & 60 min \\
\hline 45 & 0.02 & 0.02 & 0.02 & 0.01 & 0.03 & 0.02 \\
\hline 40 & 0.02 & 0.03 & 0.02 & 0.02 & 0.03 & 0.04 \\
\hline 35 & 0.04 & 0.03 & 0.05 & 0.02 & 0.05 & 0.04 \\
\hline 30 & 0.18 & 0.16 & 0.19 & 0.1 & 0.11 & 0.19 \\
\hline 25 & 0.64 & 0.68 & 0.72 & 0.52 & 0.54 & 0.68 \\
\hline 20 & 2.78 & 2.7 & 2.72 & 2.37 & 2.6 & 2.7 \\
\hline 15 & 9.2 & 9.59 & 9.37 & 9.24 & 9.06 & 9.0 \\
\hline 10 & 23.37 & 24.31 & 23.93 & 25.07 & 23.57 & 26.56 \\
\hline 5 & 30.86 & 26.74 & 26.22 & 30.53 & 29.47 & 24.2 \\
\hline Non-mag & 32.89 & 35.74 & 36.76 & 32.11 & 34.55 & 36.58 \\
\hline
\end{tabular}

Rotation separation

\begin{tabular}{|c|c|c|c|c|c|c|}
\hline \multirow[t]{3}{*}{ Fraction } & \multicolumn{6}{|c|}{ Separated weight percent } \\
\hline & \multicolumn{3}{|c|}{ With magnetic treatment } & \multicolumn{3}{|c|}{ No magnetic treatment } \\
\hline & $15 \mathrm{~min}$ & $30 \mathrm{~min}$ & $60 \mathrm{~min}$ & $15 \mathrm{~min}$ & $30 \mathrm{~min}$ & $60 \mathrm{~min}$ \\
\hline $\begin{array}{l}+25 \mathrm{~mm} \text { lift } \\
15-25 \mathrm{~mm} \text { lift } \\
\text { Rotate } \\
\text { Rot-low mag } \\
\text { Non-rotate }\end{array}$ & $\begin{array}{c}0.9 \\
11.98 \\
22.15 \\
10.95 \\
54.02\end{array}$ & $\begin{array}{l}0.92 \\
12.29 \\
22.36 \\
11.23 \\
53.2\end{array}$ & $\begin{array}{c}1.0 \\
12.09 \\
24.56 \\
12.38 \\
49.96\end{array}$ & $\begin{array}{r}0.67 \\
11.61 \\
23.39 \\
10.95 \\
53.37\end{array}$ & $\begin{array}{r}0.76 \\
11.66 \\
24.24 \\
11.16 \\
52.18\end{array}$ & $\begin{array}{l}0.97 \\
11.7 \\
24.78 \\
10.95 \\
51.6\end{array}$ \\
\hline
\end{tabular}

TABLE IV Separation results for the $400^{\circ} \mathrm{C}$ roast

\begin{tabular}{|c|c|c|c|c|c|c|}
\hline \multicolumn{7}{|c|}{ RMF lift separation } \\
\hline \multirow{3}{*}{$\begin{array}{l}\text { Separation } \\
\text { distance }(\mathrm{mm})\end{array}$} & \multicolumn{6}{|c|}{ Separated weight percent } \\
\hline & \multicolumn{3}{|c|}{ With magnetic treatment } & \multicolumn{3}{|c|}{ No magnetic treatment } \\
\hline & $15 \min$ & $30 \mathrm{~min}$ & $60 \min$ & $15 \mathrm{~min}$ & $30 \mathrm{~min}$ & $60 \mathrm{~min}$ \\
\hline 45 & 0.01 & 0.0 & 0.01 & 0.01 & 0.01 & 0.01 \\
\hline 40 & 0.03 & 0.03 & 0.02 & 0.02 & 0.03 & 0.02 \\
\hline 35 & 0.05 & 0.03 & 0.04 & 0.05 & 0.03 & 0.04 \\
\hline 30 & 0.13 & 0.08 & 0.08 & 0.12 & 0.09 & 0.09 \\
\hline 25 & 0.43 & 0.49 & 0.47 & 0.43 & 0.39 & 0.53 \\
\hline 20 & 1.68 & 1.86 & 2.25 & 1.73 & 1.61 & 2.0 \\
\hline 15 & 6.29 & 9.92 & 7.89 & 6.18 & 6.54 & 7.63 \\
\hline 10 & 17.7 & 19.23 & 20.36 & 18.42 & 19.39 & 20.27 \\
\hline 5 & 37.65 & 36.39 & 37.92 & 40.56 & 36.97 & 39.15 \\
\hline Non-mag & 36.04 & 34.96 & 30.97 & 32.48 & 34.94 & 30.27 \\
\hline
\end{tabular}

Rotation separation

Fraction

Separated weight percent

\begin{tabular}{lrrrrrrr} 
& \multicolumn{3}{c}{ With magnetic treatment } & & \multicolumn{3}{c}{ No magnetic treatment } \\
\cline { 2 - 3 } & $15 \mathrm{~min}$ & $30 \mathrm{~min}$ & $60 \mathrm{~min}$ & & $15 \mathrm{~min}$ & $30 \mathrm{~min}$ & $60 \mathrm{~min}$ \\
\hline$+25 \mathrm{~mm}$ lift & 0.65 & 0.63 & 0.61 & & 0.63 & 0.55 & 0.68 \\
$15-25 \mathrm{~mm}$ lift & 7.97 & 8.78 & 10.14 & & 7.91 & 8.15 & 9.63 \\
Rotate & 30.25 & 36.58 & 43.37 & & 28.35 & 37.66 & 45.35 \\
Rot-low mag & 12.2 & 15.82 & 24.83 & & 11.48 & 14.9 & 22.37 \\
Non-rotate & 48.94 & 38.19 & 21.05 & & 51.62 & 38.74 & 21.97 \\
\hline
\end{tabular}


TABLE V Separation results for the $450^{\circ} \mathrm{C}$ roast

\begin{tabular}{|c|c|c|c|c|c|c|}
\hline \multicolumn{7}{|c|}{$R M F$ lift separation } \\
\hline \multirow{3}{*}{$\begin{array}{l}\text { Separation } \\
\text { distance }(\mathrm{mm})\end{array}$} & \multicolumn{6}{|c|}{ Separated weight percent } \\
\hline & \multicolumn{3}{|c|}{ With magnetic treatment } & \multicolumn{3}{|c|}{ No magnetic treatment } \\
\hline & $15 \mathrm{~min}$ & $30 \mathrm{~min}$ & 60 min & $15 \mathrm{~min}$ & $30 \mathrm{~min}$ & $60 \mathrm{~min}$ \\
\hline 45 & 0.01 & 0.01 & 0.01 & 0.01 & 0.01 & 0.01 \\
\hline 40 & 0.04 & 0.06 & 0.11 & 0.05 & 0.1 & 0.12 \\
\hline 35 & 0.08 & 0.13 & 0.14 & 0.07 & 0.13 & 0.12 \\
\hline 30 & 0.19 & 0.26 & 0.32 & 0.18 & 0.24 & 0.24 \\
\hline 25 & 0.76 & 1.1 & 1.14 & 0.58 & 0.89 & 1.0 \\
\hline 20 & 2.64 & 4.0 & 4.21 & 2.42 & 3.66 & 3.61 \\
\hline 15 & 8.86 & 13.16 & 13.57 & 8.59 & 13.01 & 13.04 \\
\hline 10 & 27.23 & 35.3 & 37.66 & 25.4 & 37.21 & 35.0 \\
\hline 5 & 37.28 & 30.81 & 27.67 & 39.89 & 29.78 & 32.34 \\
\hline Non-mag & 22.92 & 15.17 & 15.17 & 22.81 & 14.97 & 14.51 \\
\hline \multicolumn{7}{|c|}{ Rotation separation } \\
\hline \multirow[t]{3}{*}{ Fraction } & \multicolumn{6}{|c|}{ Separated weight percent } \\
\hline & \multicolumn{3}{|c|}{ With magnetic treatment } & \multicolumn{3}{|c|}{ No magnetic treatment } \\
\hline & $15 \min$ & $30 \mathrm{~min}$ & $60 \mathrm{~min}$ & $15 \mathrm{~min}$ & $30 \min$ & $60 \mathrm{~min}$ \\
\hline$+25 \mathrm{~mm} \mathrm{lift}$ & 1.08 & 1.56 & 1.72 & 0.89 & 1.37 & 1.49 \\
\hline $15-25 \mathrm{~mm} \mathrm{lift}$ & 11.5 & 17.16 & 17.78 & 11.01 & 16.67 & 16.65 \\
\hline Rotate & 48.53 & 61.89 & 58.75 & 49.92 & 62.72 & 59.42 \\
\hline Rot-low mag & 21.77 & 14.3 & 16.22 & 23.11 & 13.82 & 16.19 \\
\hline Non-rotate & 17.17 & 5.1 & 5.52 & 15.07 & 5.42 & 6.24 \\
\hline
\end{tabular}
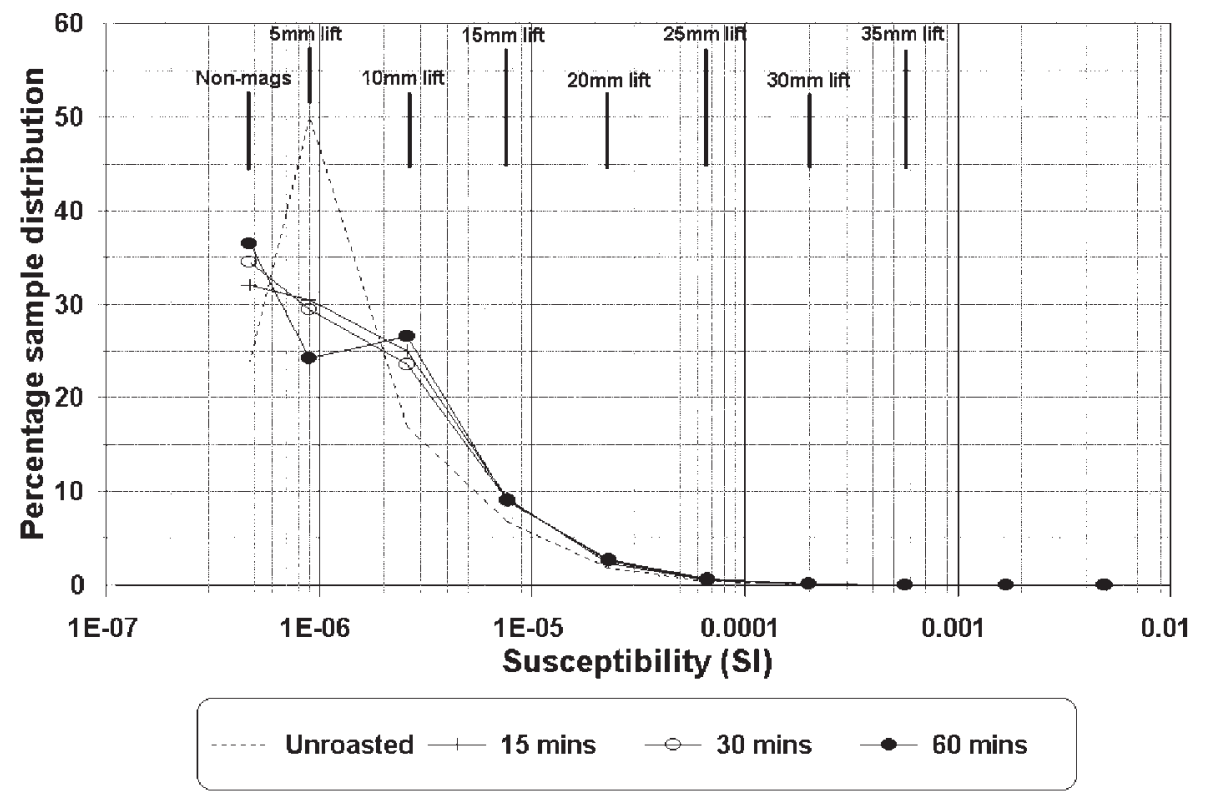

FIGURE 4 The effect of a $350^{\circ} \mathrm{C}$ roast on ilmenite magnetic susceptibility. The susceptibilities for the various lift separation distances are indicated. 
The reason why such a low temperature roast should have such an effect on magnetic susceptibility is at least partly explained in Fig. 5, which compares the $350^{\circ} \mathrm{C} 15 \mathrm{~min}$ roast results with the separation results on the demagnetised unroasted sample. The two distributions are very similar, indicating that the thermal effects of the $350^{\circ} \mathrm{C}$ roast have demagnetised the sample - removing the effects of the initial high intensity (WHIMS) separation mentioned above.

Some interesting points arise from the data in Fig. 5. One is that the initial $10 \mathrm{~mm}$ lift separation of the unroasted sample (in a rotating field of about $0.2 \mathrm{~T}$ ) had not demagnetised the remaining particles, most of which would not be able to rotate at this field. This indicates quite a high coercive force. Another is that some particles appear to have become more magnetic under the effects of both magnetic and thermal demagnetisation.

The decrease in magnetic susceptibility caused by both magnetic and thermal demagnetisation can be understood as a randomisation of high-coercivity domain orientations, but there remains the apparent increase in magnetisation shown by some other particles. Why should some particles decrease their magnetisation in a magnetising field (the initial WHIMS separation), but increase it under both thermal and magnetic demagnetising conditions? Cooling the roasted sample in a magnetic field has little effect on these particles.

In comparison, particle rotation is little affected at $350^{\circ} \mathrm{C}$. There is a marginal increase in particle rotation, with no indication that some particles may have lost rotation. The large increase in the $10 \mathrm{~mm}$ lift fraction, and even the $15 \mathrm{~mm}$ lift fraction in Fig. 5, has not translated into an equivalent increase in particle rotation. There has been an increase in susceptibility without the expected increase in rotatability.

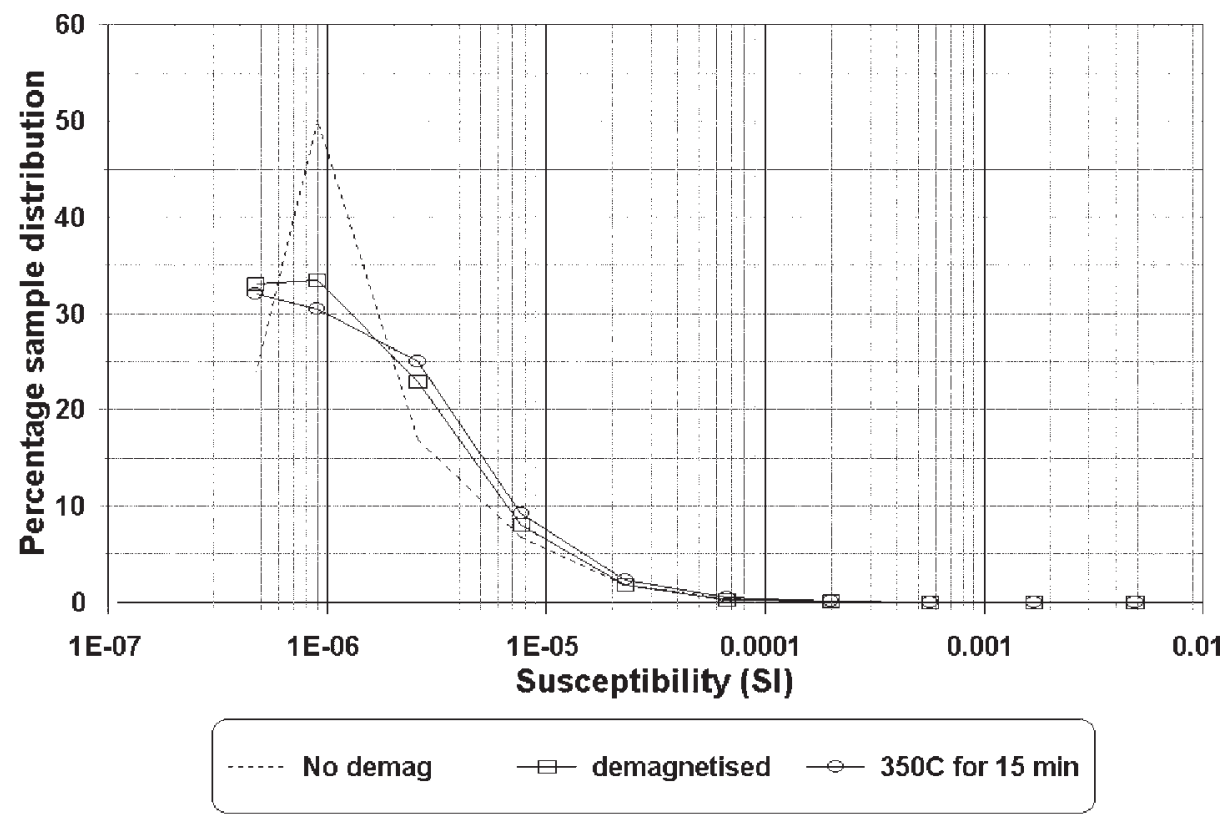

FIGURE 5 The effect of demagnetising the unroasted ilmenite, compared to the effect of a $350^{\circ} \mathrm{C}$ roast for $15 \mathrm{~min}$. The demagnetisation was carried out in a $0.4 \mathrm{~T}$ field rotating at $170 \mathrm{~Hz}$. 
Cooling the sample in a magnetic field produced no identifiable effect on particle rotation. Thus all six separations, over three roasted samples at this temperature, produced similar results for both lift and rotation separation.

\section{2. $400^{\circ} \mathrm{C}$}

Figure 6 compares the results for the 350 and $400^{\circ} \mathrm{C} 30$ min roasts. Roast times from 15 to 60 min produce almost identical results. The particles that lost susceptibility at $350^{\circ} \mathrm{C}$ have remained unchanged at $400^{\circ} \mathrm{C}$, but those particles that showed an increase in susceptibility at $350^{\circ} \mathrm{C}$ have had it reversed at $400^{\circ} \mathrm{C}$. Cooling the sample in a magnetic field had no measurable effect on the $10 \mathrm{~mm}$ lift fractions or above, and only for the 15 min roast did magnetic cooling have any effect at all, when it appears to have shifted a small percentage of particles from the $5 \mathrm{~mm}$ lift fraction down to the non-magnetic fraction.

In definite contrast, particle rotation begins to increase very significantly at $400^{\circ} \mathrm{C}$, as shown in Fig. 7 - apparently with no associated increase in particle susceptibility. This is the opposite to what happened at $350^{\circ} \mathrm{C}$.

\section{3. $450^{\circ} \mathrm{C}$}

At $450^{\circ} \mathrm{C}$ the magnetic susceptibility begins to increase across the whole sample (Fig. 8). The non-magnetic fraction, for the $30 \mathrm{~min}$ roast, reaches it lowest value for the whole range of roast temperatures. A $30 \mathrm{~min}$ roast seems sufficient to cause nearly all the magnetic susceptibility change that can occur at this temperature, but a $15 \mathrm{~min}$ roast is not long enough.

Particle rotation, at $450^{\circ} \mathrm{C}$ (Fig. 9), shows a similar pattern of increase to that shown by susceptibility. As for susceptibility, a $30 \mathrm{~min}$ roast is required for the magnetic
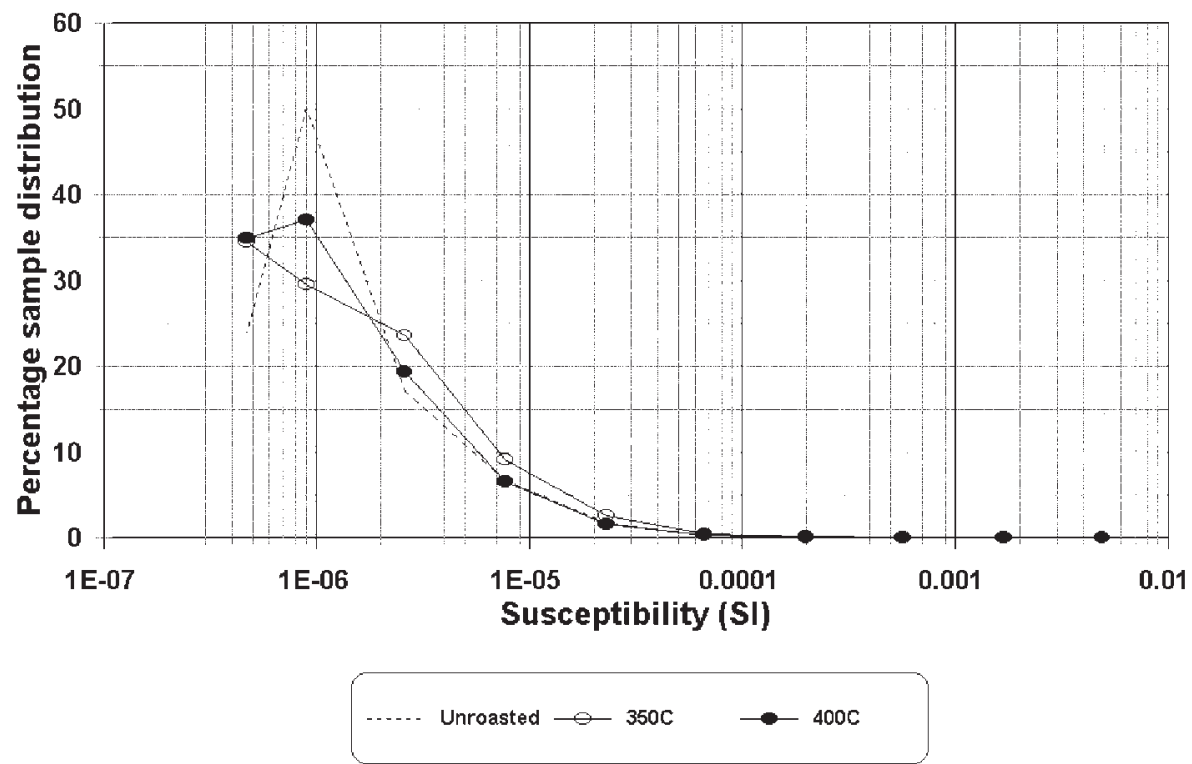

FIGURE 6 Comparison of the effects of $350^{\circ} \mathrm{C}$ and $400^{\circ} \mathrm{C}$ roasts on magnetic susceptibility. 


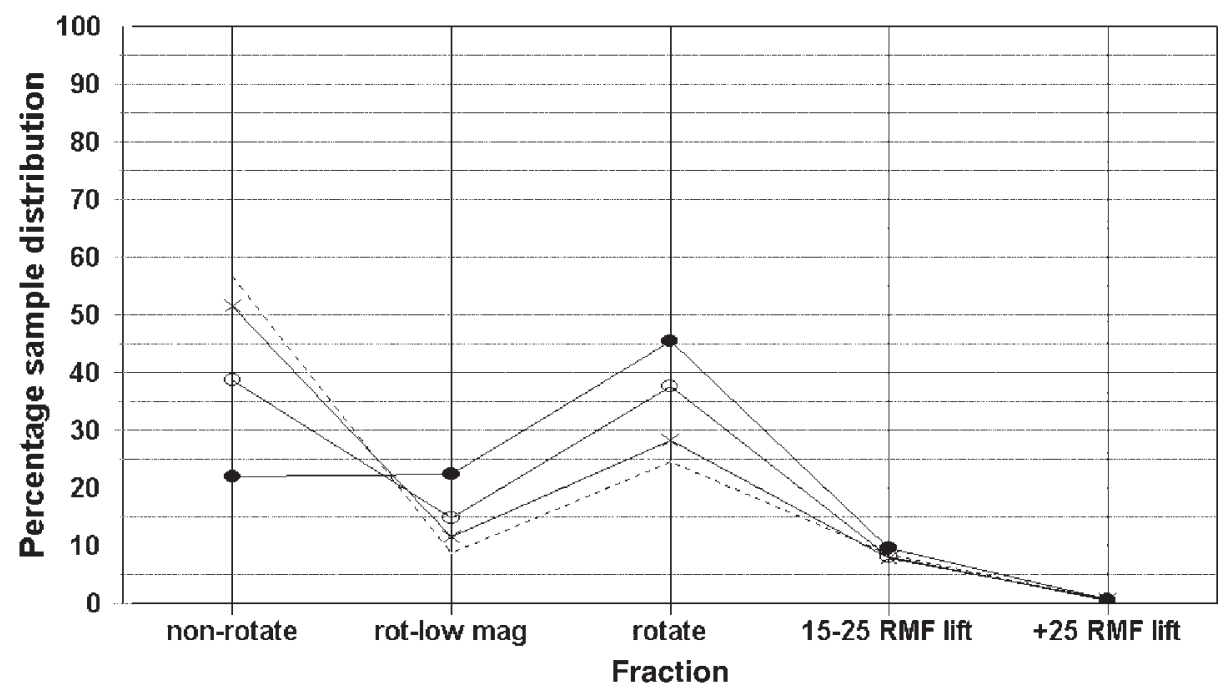

Unroasted $\longrightarrow 15$ mins $\multimap 30$ mins $\rightarrow-60$ mins

FIGURE 7 The effect of a $400^{\circ} \mathrm{C}$ roast on particle rotation, showing the effect of roast time.

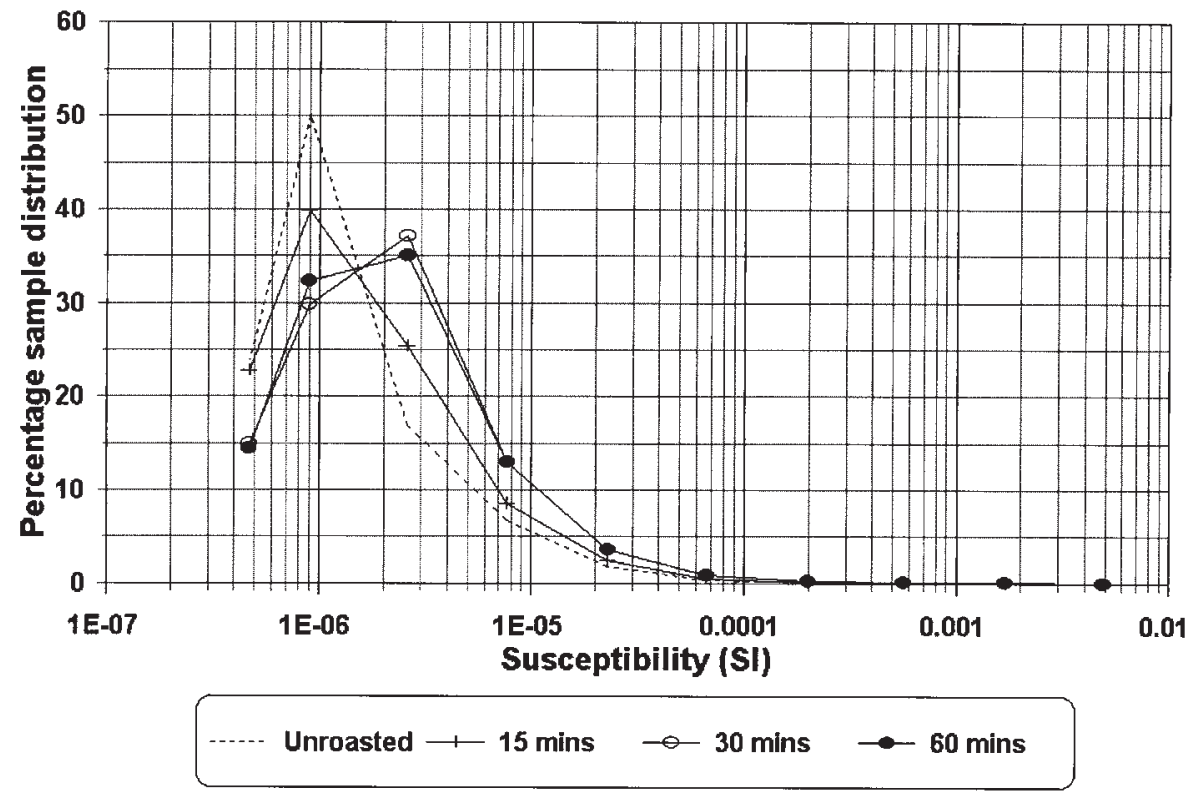

FIGURE 8 The effect of a $450^{\circ} \mathrm{C}$ roast on magnetic susceptibility, showing the effect of roast time.

changes to completely penetrate the particles, and further roast time appears to be just wasted energy. At $450^{\circ} \mathrm{C}$ the increases in particle rotation are accompanied by increases in particle susceptibility. This is what normally happens.

By $450^{\circ} \mathrm{C}$ the non-mag and non-rotate fractions have both reached their minimum. 


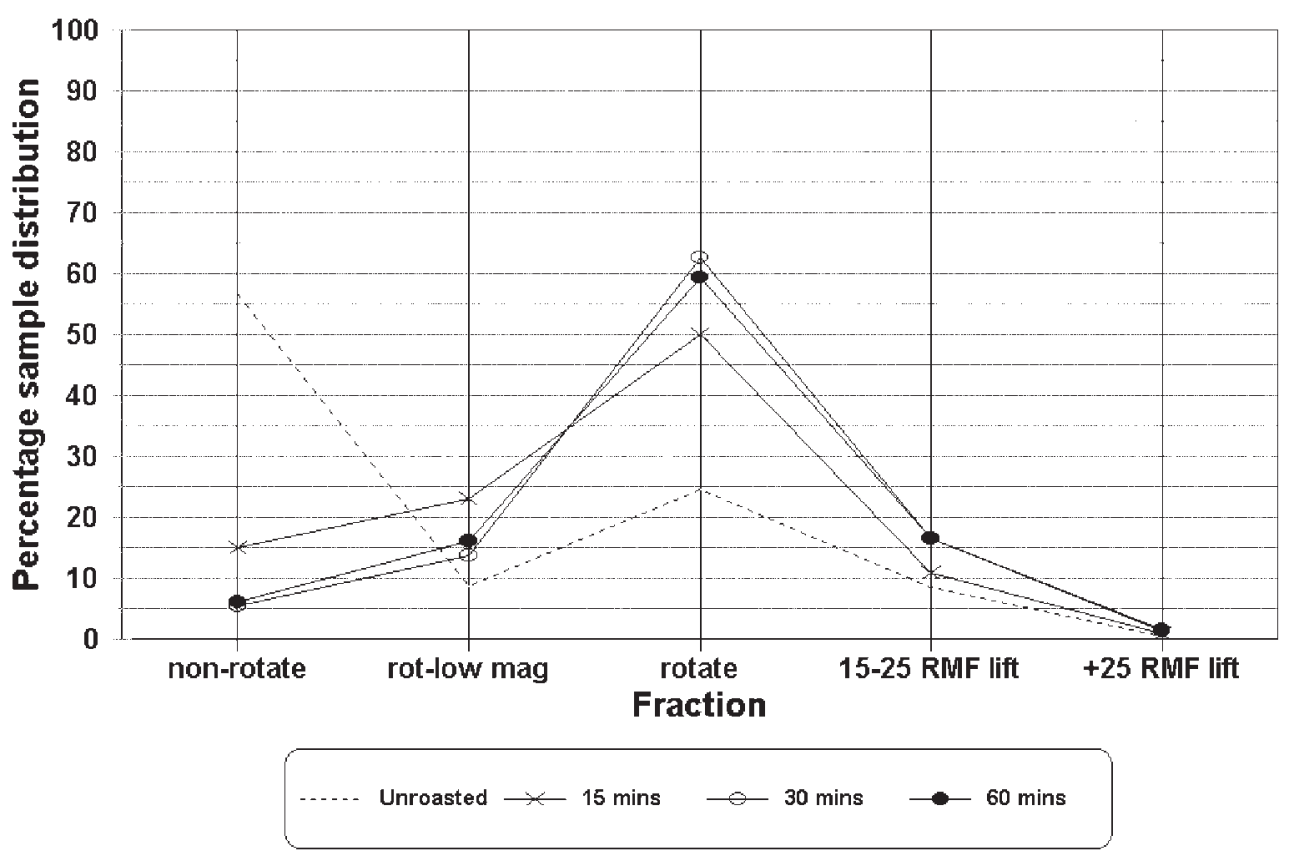

FIGURE 9 The effect of a $450^{\circ} \mathrm{C}$ roast on particle rotation, showing the effect of roast time.

\section{4. $450-650{ }^{\circ} \mathrm{C}$}

The separation fraction percentages for the $450-650^{\circ} \mathrm{C}$ roasts are given in Tables VI-IX.

Above $450^{\circ} \mathrm{C}$ susceptibility, and rotatability so far as can be determined, increase in a fairly 'linear' way with increasing roast temperatures, as illustrated in Figs. 10 and 11. However, while most particles are increasing in both susceptibility and rotatability, increasing numbers of particles are decreasing equally in both these properties. This effect can also be seen in Figs. 10 and 11.

At $650^{\circ} \mathrm{C}$ this trend is increasing, implying a limit to the usefulness of oxidising magnetising roasts at somewhere between 700 and $800^{\circ} \mathrm{C}$, at least for this particular sample.

Up to $500^{\circ} \mathrm{C}$, cooling the sample in a magnetic field has little effect on either particle susceptibility or particle rotatability, but at $550^{\circ} \mathrm{C}$ this changes and the magnetic field cooling further increases susceptibility, as shown in Fig. 12. It is not determined whether particle rotation also increases, because the rotation separations used here determine whether or not rotation sufficient for separation occurs, it tells nothing about the rotation strength for the more susceptible particles.

At $500^{\circ} \mathrm{C}$ changing the roast time from 15 to $60 \mathrm{~min}$ had no effect on either the susceptibility or rotation results achieved, but from $550^{\circ} \mathrm{C}$ a longer roast also produces a greater susceptibility increase. Unfortunately, as can be seen from Fig. 13, this is partially off-set by an increase in the non-magnetic fraction, as more particles become non-magnetic. Although it is not determined if the roast time at $550^{\circ} \mathrm{C}$ or above increased particle rotation, at least it did not detectably increase the non-rotating fraction. 
TABLE VI Separation results for the $500^{\circ} \mathrm{C}$ roast.

\begin{tabular}{|c|c|c|c|c|c|c|}
\hline \multicolumn{7}{|c|}{ RMF lift separation } \\
\hline \multirow[t]{3}{*}{ Separation distance $(\mathrm{mm})$} & \multicolumn{6}{|c|}{ Separated weight percent } \\
\hline & \multicolumn{3}{|c|}{ With magnetic treatment } & \multicolumn{3}{|c|}{ No magnetic treatment } \\
\hline & $15 \mathrm{~min}$ & $30 \mathrm{~min}$ & $60 \mathrm{~min}$ & $15 \mathrm{~min}$ & $30 \mathrm{~min}$ & 60 min \\
\hline 45 & 0.01 & 0.01 & 0.02 & 0.02 & 0.01 & 0.01 \\
\hline 40 & 0.1 & 0.06 & 0.06 & 0.03 & 0.06 & 0.03 \\
\hline 35 & 0.15 & 0.17 & 0.15 & 0.09 & 0.14 & 0.08 \\
\hline 30 & 0.39 & 0.42 & 0.58 & 0.28 & 0.43 & 0.33 \\
\hline 25 & 1.53 & 1.86 & 2.23 & 1.17 & 1.36 & 1.47 \\
\hline 20 & 5.77 & 7.03 & 9.18 & 5.31 & 6.14 & 6.39 \\
\hline 15 & 18.21 & 21.94 & 23.84 & 18.53 & 21.56 & 23.41 \\
\hline 10 & 45.01 & 45.14 & 41.53 & 47.61 & 46.58 & 45.59 \\
\hline 5 & 14.33 & 8.72 & 7.47 & 12.91 & 9.58 & 8.2 \\
\hline Non-mag & 14.49 & 14.65 & 14.93 & 14.05 & 14.13 & 14.48 \\
\hline
\end{tabular}

Rotation separation

\begin{tabular}{|c|c|c|c|c|c|c|}
\hline \multirow[t]{3}{*}{ Fraction } & \multicolumn{6}{|c|}{ Separated weight percent } \\
\hline & \multicolumn{3}{|c|}{ With magnetic treatment } & \multicolumn{3}{|c|}{ No magnetic treatment } \\
\hline & $15 \mathrm{~min}$ & $30 \mathrm{~min}$ & $60 \mathrm{~min}$ & $15 \mathrm{~min}$ & $30 \mathrm{~min}$ & $60 \mathrm{~min}$ \\
\hline$+25 \mathrm{~mm}$ lift & 2.18 & 2.52 & 3.04 & 1.59 & 2.0 & 1.92 \\
\hline $15-25 \mathrm{~mm}$ lift & 23.98 & 28.97 & 33.02 & 23.84 & 27.7 & 29.8 \\
\hline Rotate & 55.82 & 51.79 & 46.69 & 56.32 & 54.12 & 51.65 \\
\hline Rot-low mag & 12.23 & 11 & 11.12 & 12.81 & 10.49 & 10.53 \\
\hline Non-rotate & 5.87 & 5.72 & 6.11 & 5.44 & 5.67 & 6.08 \\
\hline
\end{tabular}

TABLE VII Separation results for the $550^{\circ} \mathrm{C}$ roast.

\begin{tabular}{|c|c|c|c|c|c|c|}
\hline \multicolumn{7}{|c|}{ RMF lift separation } \\
\hline \multirow{3}{*}{$\begin{array}{l}\text { Separation } \\
\text { distance }(\mathrm{mm})\end{array}$} & \multicolumn{6}{|c|}{ Separated weight percent } \\
\hline & \multicolumn{3}{|c|}{ With magnetic treatment } & \multicolumn{3}{|c|}{ No magnetic treatment } \\
\hline & $15 \mathrm{~min}$ & $30 \mathrm{~min}$ & $60 \mathrm{~min}$ & $15 \min$ & $30 \min$ & $60 \mathrm{~min}$ \\
\hline 45 & 0.01 & 0.01 & 0.01 & 0.01 & 0.01 & 0.0 \\
\hline 40 & 0.06 & 0.08 & 0.07 & 0.05 & 0.03 & 0.03 \\
\hline 35 & 0.16 & 0.23 & 0.24 & 0.1 & 0.08 & 0.05 \\
\hline 30 & 0.83 & 0.9 & 1.08 & 0.56 & 0.47 & 0.25 \\
\hline 25 & 2.94 & 3.27 & 5.11 & 1.66 & 1.62 & 1.9 \\
\hline 20 & 12.04 & 13.03 & 15.97 & 7.42 & 7.58 & 8.28 \\
\hline 15 & 27.3 & 29.03 & 31.17 & 25.39 & 27.45 & 30.68 \\
\hline 10 & 37.42 & 34.59 & 27.4 & 44.84 & 43.14 & 38.42 \\
\hline 5 & 4.39 & 3.53 & 2.49 & 5.45 & 4.79 & 4.19 \\
\hline Non-mag & 14.85 & 15.32 & 16.48 & 14.49 & 14.83 & 16.2 \\
\hline \multicolumn{7}{|c|}{ Rotation separation } \\
\hline \multirow[t]{3}{*}{ Fraction } & \multicolumn{6}{|c|}{ Separated weight percent } \\
\hline & \multicolumn{3}{|c|}{ With magnetic treatment } & \multicolumn{3}{|c|}{ No magnetic treatment } \\
\hline & $15 \min$ & $30 \min$ & $60 \mathrm{~min}$ & $15 \min$ & $30 \min$ & $60 \mathrm{~min}$ \\
\hline$+25 \mathrm{~mm}$ lift & 4.0 & 4.49 & 6.51 & 2.38 & 2.21 & 2.23 \\
\hline $15-25 \mathrm{~mm}$ lift & 39.34 & 42.06 & 47.14 & 32.81 & 35.03 & 38.96 \\
\hline Rotate & 41.04 & 37.1 & 28.64 & 49.29 & 46.93 & 41.88 \\
\hline Rot-low mag & 8.62 & 9.54 & 9.77 & 8.09 & 8.52 & 8.67 \\
\hline Non-rotate & 6.99 & 6.79 & 7.95 & 7.37 & 7.32 & 8.26 \\
\hline
\end{tabular}


TABLE VIII Separation results for the $600^{\circ} \mathrm{C}$ roast

\begin{tabular}{|c|c|c|c|c|c|c|}
\hline \multicolumn{7}{|c|}{$R M F$ lift separation } \\
\hline \multirow[t]{3}{*}{ Separation distance $(\mathrm{mm})$} & \multicolumn{6}{|c|}{ Separated weight percent } \\
\hline & \multicolumn{3}{|c|}{ With magnetic treatment } & \multicolumn{3}{|c|}{ No magnetic treatment } \\
\hline & $15 \mathrm{~min}$ & $30 \mathrm{~min}$ & $60 \mathrm{~min}$ & $15 \min$ & $30 \mathrm{~min}$ & $60 \mathrm{~min}$ \\
\hline 45 & 0.02 & 0.01 & 0.02 & 0.01 & 0.0 & 0.01 \\
\hline 40 & 0.06 & 0.1 & 0.07 & 0.03 & 0.02 & 0.03 \\
\hline 35 & 0.32 & 0.37 & 0.45 & 0.05 & $0.0 \overline{6}$ & 0.05 \\
\hline 30 & 1.15 & 1.55 & 2.06 & 0.31 & 0.38 & 0.46 \\
\hline 25 & 4.37 & 5.6 & 7.52 & 1.84 & 2.41 & 2.63 \\
\hline 20 & 14.63 & 17.12 & 19.24 & 7.48 & 9.41 & 10.72 \\
\hline 15 & 30.62 & 31.9 & 32.23 & 30.92 & 33.82 & 38.05 \\
\hline 10 & 29.6 & 23.63 & 18.31 & 38.89 & 32.14 & 26.76 \\
\hline 5 & 3.58 & 3.24 & 2.27 & 5.16 & 5.57 & 3.69 \\
\hline Non-mag & 15.65 & 16.47 & 17.83 & 15.32 & 16.19 & 17.61 \\
\hline
\end{tabular}

Rotation separation

\begin{tabular}{|c|c|c|c|c|c|c|}
\hline \multirow[t]{3}{*}{ Fraction } & \multicolumn{6}{|c|}{ Separated weight percent } \\
\hline & \multicolumn{3}{|c|}{ With magnetic treatment } & \multicolumn{3}{|c|}{ No magnetic treatment } \\
\hline & $15 \mathrm{~min}$ & $30 \mathrm{~min}$ & $60 \mathrm{~min}$ & $15 \mathrm{~min}$ & $30 \mathrm{~min}$ & $60 \mathrm{~min}$ \\
\hline$+25 \mathrm{~mm}$ lift & 5.92 & 7.63 & 10.12 & 2.24 & 2.87 & 3.18 \\
\hline $15-25 \mathrm{~mm}$ lift & 45.25 & 49.02 & 51.47 & 38.4 & 43.23 & 48.77 \\
\hline Rotate & 32.48 & 27.17 & 20.67 & 43.39 & 37.25 & 30.79 \\
\hline Rot-low mag & 8.71 & 8.22 & 10.16 & 8.0 & 8.34 & 8.3 \\
\hline Non-rotate & 7.64 & 7.95 & 7.58 & 7.97 & 8.31 & 8.97 \\
\hline
\end{tabular}

TABLE IX Separation results for the $650^{\circ} \mathrm{C}$ roast

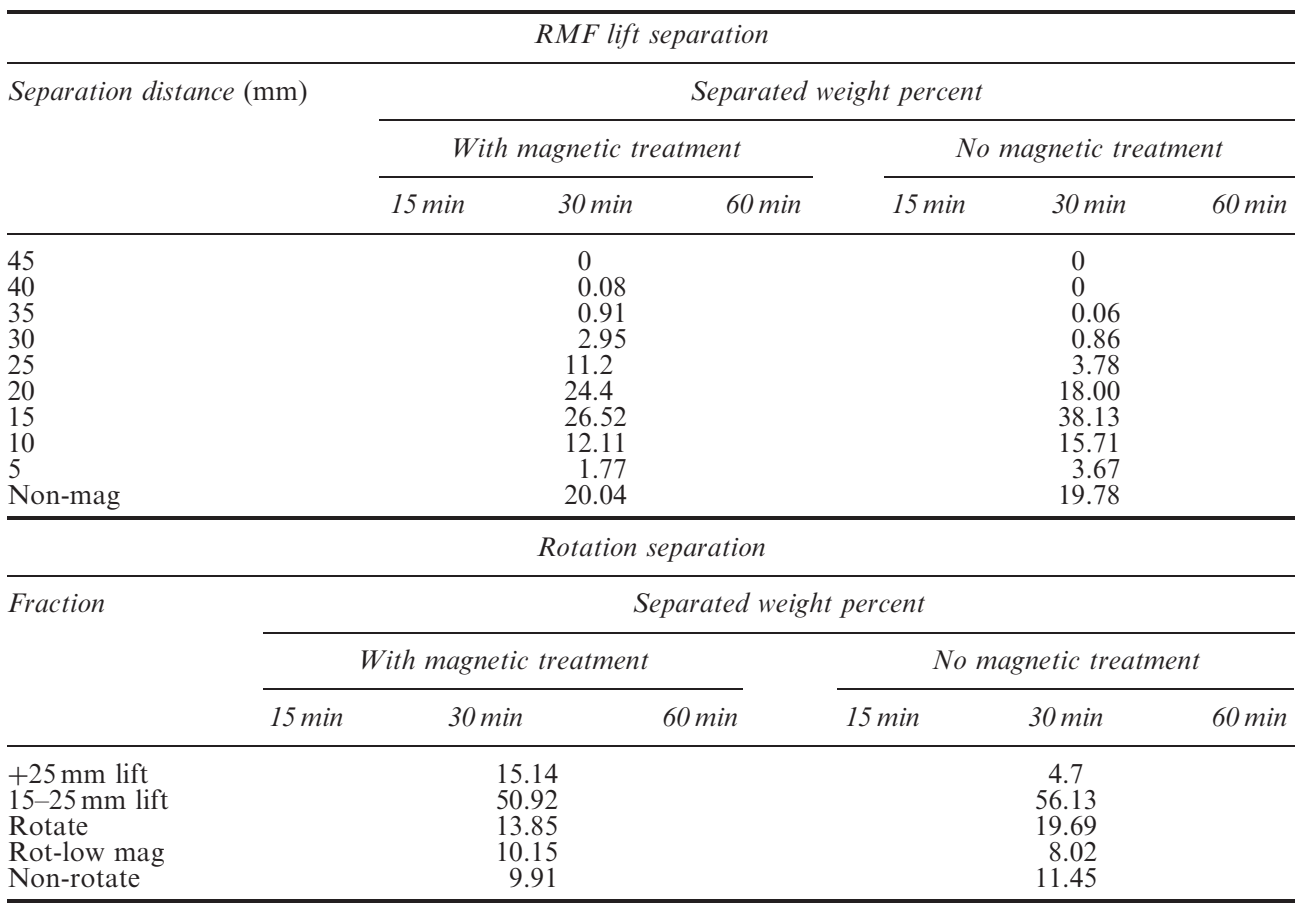




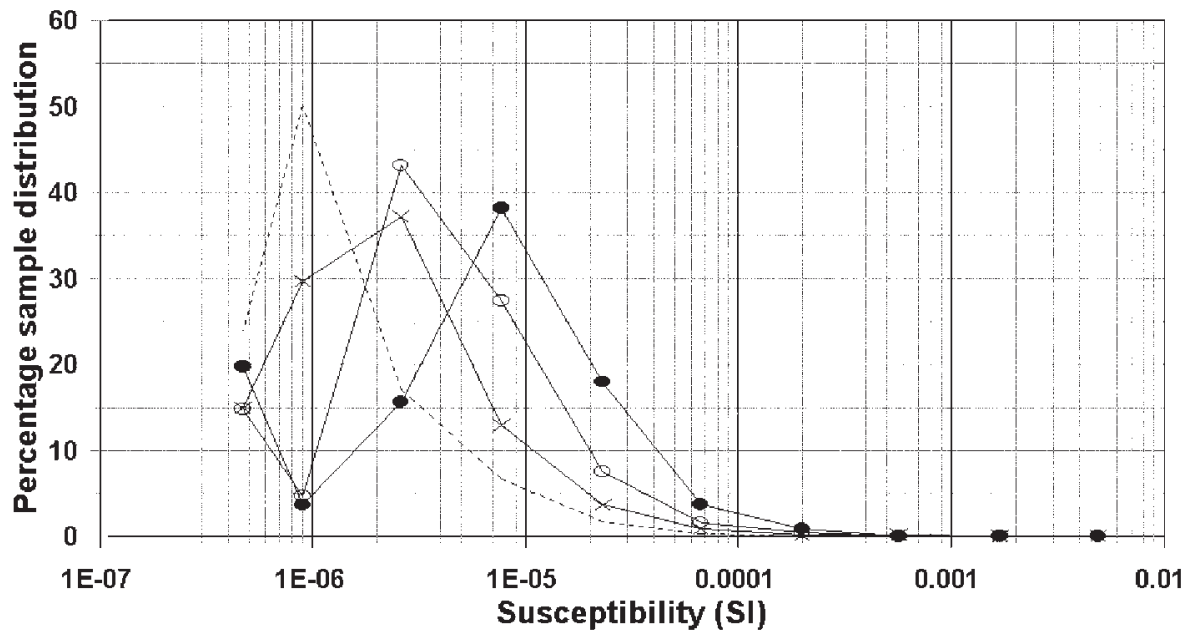

Unroasted $\longrightarrow 450 \mathrm{C}-650 \mathrm{C}-650 \mathrm{C}$

FIGURE 10 Susceptibility increases between $450^{\circ} \mathrm{C}$ and $650^{\circ} \mathrm{C}$.

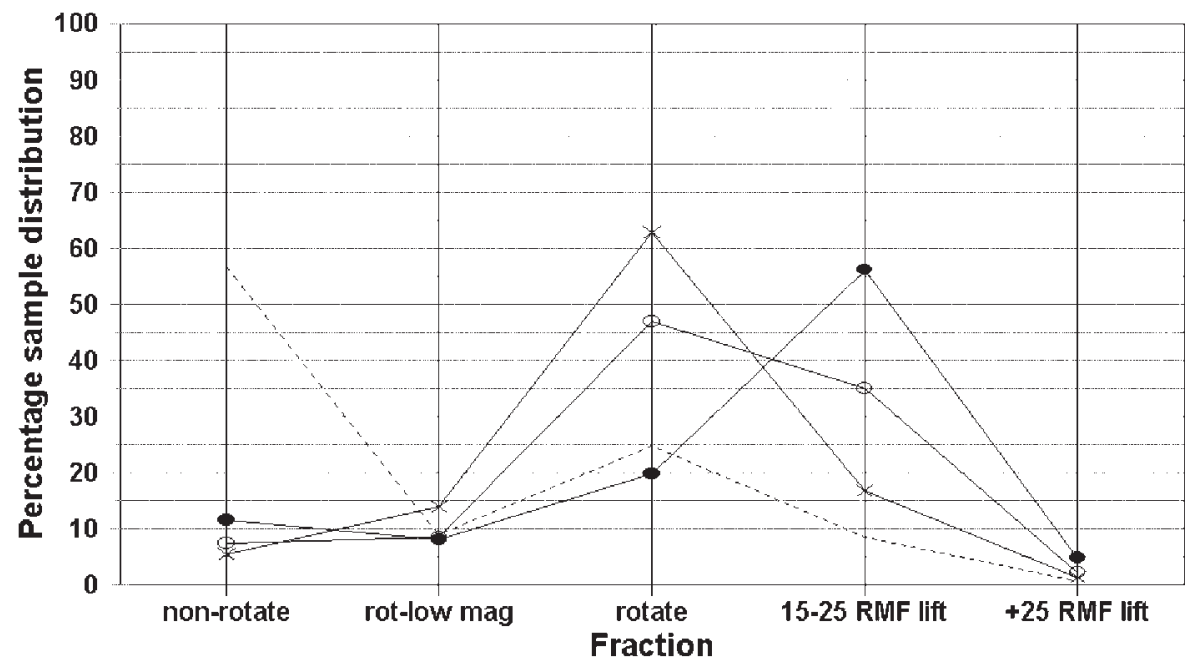

Unroasted $\longrightarrow 450 \mathrm{C} \longrightarrow 550 \mathrm{C} \longrightarrow 650 \mathrm{C}$

FIGURE 11 Rotation separation changes between $450^{\circ} \mathrm{C}$ and $650^{\circ} \mathrm{C}$.

\subsection{The Effect of Demagnetisation on Higher Temperature Roasts}

For roast temperatures above $500^{\circ} \mathrm{C}$, demagnetisation, using the method described earlier, causes a decrease in sample susceptibility. The effect of demagnetisation on the $600^{\circ} \mathrm{C} 60 \mathrm{~min}$ roast, for example, has been to reduce the sample susceptibility to approximately that of the $30 \mathrm{~min}$ roast. 


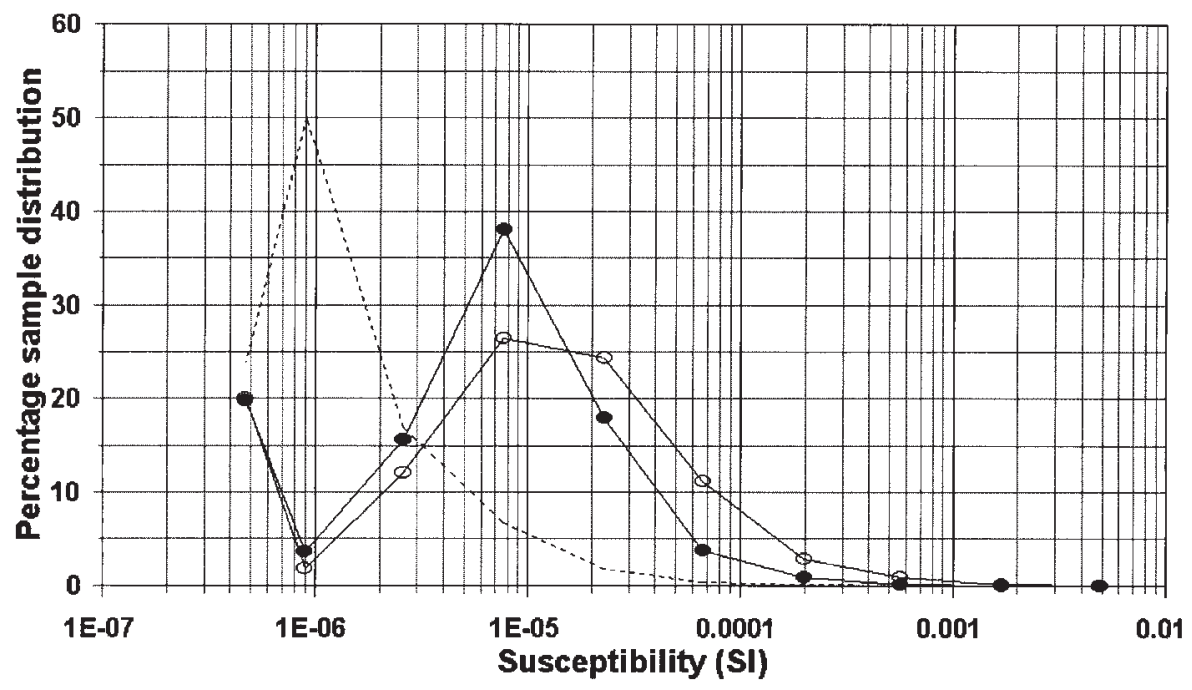

\section{Unroasted $\quad-0$ Mag treated $\quad-$ Not mag treated}

FIGURE 12 The effect of cooling the sample in a magnetic field, for a $650^{\circ} \mathrm{C} 30 \mathrm{~min}$ roast.

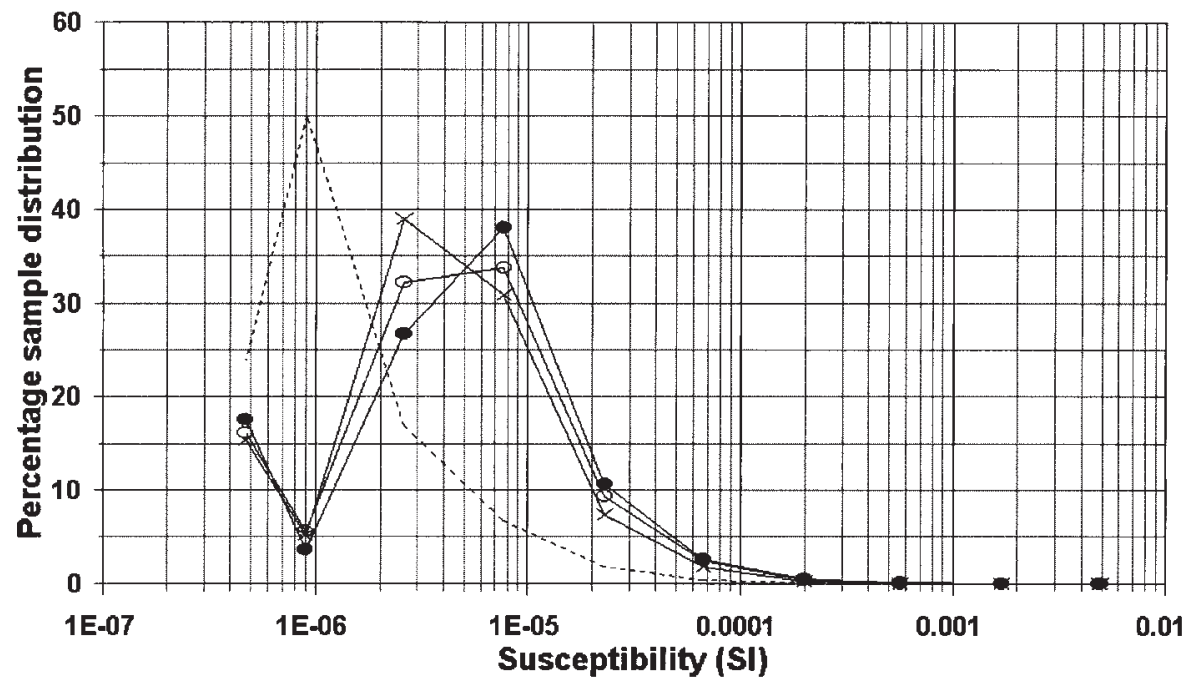

Unroasted $\multimap 15$ mins $\odot 30$ mins $\multimap 60$ mins

FIGURE 13 The effect of roast time on a $600^{\circ} \mathrm{C}$ roast.

\section{COMMENTS}

The results and comments here are based on an oxidising roast, in air, on one example of mixed Murray Basin ilmenite. Other roast atmospheres and other samples may be expected to produce slightly different results. 
The development of roast-induced magnetisation in ilmenite can be divided into three main stages:

1. Up to $450^{\circ} \mathrm{C}$. In this range, or at least between 350 and $450^{\circ} \mathrm{C}$, the particle magnetisation appears to be going through some initial changes involving independent changes in susceptibility and rotatability.

2. $500-550^{\circ} \mathrm{C}$. Susceptibility and rotatability are increasing in this range, but roast times between 15 and 60 min produce no convincing differences in the results, and cooling the sample in a magnetic field has no convincing effect.

3. $550^{\circ} \mathrm{C}$ and above. In this range most particles increase in susceptibility, but an increasing number of particles go the other way and decrease in susceptibility. Increasing the roast time and cooling the sample in a magnetic field both increase overall susceptibility, but also cause more particles to lose susceptibility. At somewhere between 700 and $800^{\circ} \mathrm{C}$, at least for the sample used here, the measured trends indicate that the decreases in susceptibility should outweigh any increases.

\subsection{0-450 ${ }^{\circ}$ : The Initial Development Stage}

The initial heating of the ilmenite to a temperature of $350^{\circ} \mathrm{C}$ appears to remove any remanent magnetism from a previous high intensity separation. Thus the previous magnetic processing history of the sample is not going to significantly affect the roast results. The fact that a remanence occurs indicates the presence of ferromagnetism (or more strictly, ferrimagnetism here). However, the fact that the remanence was not removed from the initial (i.e. not demagnetised) unroasted sample by the rotating field separation at $10 \mathrm{~mm}$ (approx $0.2 \mathrm{~T}$ ) implies quite a high coercive force for the magnetisation that has produced the remanence.

While the removal of a remanent magnetisation by the application of thermal or magnetic demagnetisation presents no conceptual problems, the observed increase in magnetisation after both thermal and magnetic demagnetisation is more difficult to understand. Even though this increase occurs at quite high susceptibilities $(10,15$ and even $20 \mathrm{~mm}$ lift in this case), where most particles would be expected to have good rotatabilities, there has not been an associated increase in particle rotation. This implies a paramagnetic nature for the susceptibility increase. Perhaps the observed magnetisation increase can be seen in one of two ways; either as being caused by the (thermal or magnetic) demagnetising event, or as being controlled by the remanent magnetism from the initial WHIMS separation. Its apparent paramagnetic nature argues against it being caused by the demagnetisation. Thus we are left with what must be very small, apparently paramagnetic, elements within a basically paramagnetic or weak ferrimagnetic particle, which carry significant magnetism that can be decreased by the presence of a surrounding remanent magnetism or increased by a demagnetisation. One explanation for such elements would be that they are superparamagnetic. Superparamagnetic elements show a very high susceptibility at the onset of superparamagnetism, but the point of onset depends on many factors, such as size, temperature, and maybe in this case, the presence or absence of a remanent magnetism field. Superparamagnetism has been identified in ilmenite-hematite by others [3], but has been associated only with low $\mathrm{Fe}_{2} \mathrm{O}_{3}$ content at temperatures well below room temperature. 
Therefore, at $350^{\circ} \mathrm{C}$, nothing has really changed in the ilmenite particles except for the removal of any remanent magnetism and its effects, but at $400^{\circ} \mathrm{C}$ we see a change towards increasing particle rotation accompanied by a puzzling reduction in particle magnetisation. This reduction is roughly equal in significance to the 'superparamagnetic' effect described above at $350^{\circ} \mathrm{C}$. A reduction in magnetisation accompanied by an increase in magnetic anisotropy would fit with a slight increase in size or change in shape for a superparamagnetic element, sufficient to move it from the superparamagnetic state to a more stable monodomain state. At just above the temperature at which it is initiated, a change in size or shape for many small magnetic elements would be expected to take a finite time to reach throughout the whole ilmenite grain, or to take different times for different grain sizes in the sample. This could explain the significantly greater effects of longer roast times for both susceptibility and rotatability between 400 and $450^{\circ} \mathrm{C}$.

Thus the magnetic changes up to $450^{\circ} \mathrm{C}$ can be seen as associated with slight changes in size or shape for very small magnetic elements within the ilmenite that appear to be small enough to exhibit superparamagnetic behaviour. By $450^{\circ} \mathrm{C}$ any evidence of superparamagnetic behaviour has ceased, but these small elements could well act as 'seeds' for the later developments in particle magnetism.

\section{2. $500^{\circ} \mathrm{C}$ : The Intermediate Stage}

At $500^{\circ} \mathrm{C}$, both cooling the roasted sample in a low magnetic field (approx $0.15 \mathrm{~T}$ ) and increasing the roast time have little effect. The non-magnetic and non-rotating fractions have remained at the minimum they reached at $450^{\circ} \mathrm{C}$, but for the more magnetic particles, susceptibility and rotation have both increased from $450^{\circ} \mathrm{C}$.

Obviously some growth of the magnetic elements within the ilmenite is going on, but it is no longer showing up in the roast time variation - indicating only that it is completed in less than $15 \mathrm{~min}$.

Most particles in the sample will be above their Curie temperature at $500^{\circ} \mathrm{C}$, and it may reasonably be expected that cooling through the Curie temperature in a magnetic field would magnetically align some of the high-coercivity elements and cause an increase in the measured susceptibility. However, it did take some $10 \mathrm{~s}$ or so to transfer the particles from the fluid-bed roast situation to the cooling trough in the magnetic field, and in the process they were allowed to fall about $50 \mathrm{~mm}$ through room-temperature air. Thus, by the time the particles had been transferred to the magnetic field most particles may well have cooled below their Curie temperatures.

A roast temperature around $500^{\circ} \mathrm{C}$ appears as steady development stage for the type of magnetic element growth that began about $450^{\circ} \mathrm{C}$.

\subsection{0-650 ${ }^{\circ}$ : The Constant Change Stage}

From 550 to $650^{\circ} \mathrm{C}$ a different type of magnetic element growth takes over, where once again longer roast times produce greater susceptibility increases. This implies a timedependent growth of the magnetic elements within or around the ilmenite grains, that is accelerated by increasing the temperature. Over the temperature range there is a fairly constant increase in sample magnetic susceptibility, accompanied by an accelerating increase in the number of particles moving down to both a low magnetic susceptibility and rotatability. Cooling the sample in a magnetic field now causes an 
increase in particle attractability, and has approximately the same effect as doubling the roast time. While a longer roast time further increases the particles moving to lower susceptibilities, cooling in a magnetic field has little effect on this tendency. A demagnetisation at $0.4 \mathrm{~T}$ does decrease measured sample susceptibility, by almost the same amount as halving the roast time (i.e. it has a similar, but opposite, effect to cooling in a magnetic field).

Exactly what is happening to particle rotation for the more magnetic particles in this temperature range is not clear, because the separation method used here will only determine whether or not particles can rotate sufficient for separation. It does not classify rotation strength for the more magnetic particles.

For temperatures of $550^{\circ} \mathrm{C}$ and above, most of the ilmenite (or ilmenite-hematite) will be above its Curie temperature, and now most of it will probably still be above the Curie temperature by the time it is placed in the magnetic field to cool. Therefore, especially if any of the magnetic 'elements' have high coercive force, cooling through the Curie or Neel temperatures in a magnetic field would be expected to cause a permanent increase in susceptibility.

The fact that demagnetisation has caused a lowering of susceptibility in samples that have not cooled in a magnetic field is a little puzzling. It would seem to indicate the destruction of some magnetic alignments between elements in the ilmenite that formed as the ilmenite cooled through the Curie temperature. If this is so, then it would fit well with the effect of cooling in a magnetic field, which would be expected to enhance such alignments.

The movement of some particles towards both a lower susceptibility and a lower rotatability looks very much like the appearance of some antiferromagnetic ordering. This would require the local growth (or coalescing) of wider hematite regions, where shape anisotropy is reduced.

\section{CONCLUSION}

The combination of the magnetic attraction and rotation separations, described here, provide many new clues to the low-temperature development of roasted ilmenite magnetic properties. However, the interpretation of these clues, attempted here, and the development of a more detailed and certain picture, requires much more examination of the problem yet.

The overall picture that emerges here for the development of magnetic properties in ilmenite roasted between 350 and $650^{\circ} \mathrm{C}$, is a gradual growth of small magnetic elements (distinct magnetic regions) within a particle that begins as basically paramagnetic at room temperature. The magnetic elements appear to begin as sufficiently small to exhibit superparamagnetic properties, and then to develop through stable singledomain to multi-domain size. This growth appears to take place in two stages, which probably represent two different growth mechanisms. The first stage, to $450^{\circ} \mathrm{C}$, appears to consist of a magnetic element growth, based on very small initially superparamagnetic-sized elements, essentially in isolation from other small magnetic elements within the particle. This stage sees the extensive development of particle rotation, but only the commencement of susceptibility increase. The second stage, above $550^{\circ} \mathrm{C}$, shows a steady increase of magnetic susceptibility. It also shows evidence of increasing magnetic interaction between magnetic elements, and possible convergence 
or combination of magnetic elements. For an increasing number of particles there appears to be a coalescing of these elements as temperature increases, to the extent that antiferromagnetic ordering begins to appear, resulting in a decrease in both susceptibility and particle rotation. Different particles would obviously commence at different points along this development, and it is possible that different regions of the one particle will be at different stages.

Particle rotation, for the ilmenite sample here, begins to increase for roasts of $400^{\circ} \mathrm{C}$. This is about $50^{\circ} \mathrm{C}$ before the point where susceptibility shows any increase, and this early change in rotatability fits with a change in the small magnetic elements from an apparently superparamagnetic behavior to a stable monodomain behavior. This implies that, for low-temperature roasting, the optimum roast conditions for the magnetic rotation separation of ilmenite will be about $50^{\circ} \mathrm{C}$ below the optimum roast conditions for conventional attraction separation.

\section{References}

[1] N.R. Allen and H. Aral, Application of rotating magnetic field (RMF) to the separation of roasted ilmenite. Proc. Int. Heavy Minerals Conference 2001, 2001, The Australasian Institute of Mining and Metallurgy, Melbourne, pp. 193-200.

[2] N.R. Allen, Mineral particle rotation measurements for magnetic rotation separation. Magn. Electr. Sep., 11(3) (2002), 155-168.

[3] N.E. Brown, A. Navrotsky, G.L. Nord, Jr. and S.K. Banergee, Hematite-ilmenite $\left.\left(\mathrm{Fe}_{2} \mathrm{O}_{3}-\mathrm{FeTiO}\right)_{3}\right)$ solid solutions: determination of Fe-Ti order from magnetic properties. American Mineralogist, 78 (1993), 941-951. 